\title{
The Effect of 6-Week Combined Balance and Plyometric Training on Change of Direction Performance of Elite Badminton Players
}

\author{
Zhenxiang Guo ${ }^{1,2 \dagger}$, Yan Huang ${ }^{3 \dagger}$, Zhihui Zhou ${ }^{1}$, Bo Leng ${ }^{1}$, Wangcheng Gong ${ }^{4}$, \\ Yixiong Cui ${ }^{5 *}$ and Dapeng Bao ${ }^{4 *}$
}

${ }^{1}$ Sports Coaching College, Beijing Sport University, Beijing, China, ${ }^{2}$ Department of Physical Education, Nanjing University of Aeronautics and Astronautics, Nanjing, China, ${ }^{3}$ Beijing Research Institute of Sports Science, Beijing, China, ${ }^{4}$ China Institute of Sport and Health Science, Beijing Sport University, Beijing, China, ${ }^{5}$ Al Sports Engineering Lab, School of Sports Engineering, Beijing Sport University, Beijing, China

\section{OPEN ACCESS}

Edited by:

José Luis Losada,

University of Barcelona, Spain

Reviewed by:

Francesco Fischetti,

University of Bari Aldo Moro, Italy

Ana Ruivo Alves,

Polytechnic Institute of Beja, Portugal

Yassine Negra,

University of Manouba, Tunisia

*Correspondence:

Dapeng Bao

baodp@bsu.edu.cn

Yixiong Cui

cuiyixiong@bsu.edu.cn

tThese authors have contributed equally to this work

Specialty section:

This article was submitted to

Movement Science and Sport

Psychology,

a section of the journal

Frontiers in Psychology

Received: 24 March 2021

Accepted: 10 May 2021

Published: 10 June 2021

Citation:

Guo Z, Huang Y, Zhou Z, Leng B, Gong W, Cui $Y$ and Bao D (2021) The Effect of 6-Week Combined Balance and Plyometric Training on Change of

Direction Performance of Elite Badminton Players.

Front. Psychol. 12:684964.

doi: 10.3389/fpsyg.2021.684964
The study aimed to investigate the effect of combined balance and plyometric training on the change of direction (COD) performance of badminton athletes. Sixteen elite male badminton players volunteered to participate and were randomly assigned to a balance-plyometric group (BP: $n=8$ ) and plyometric group (PL: $n=8)$. The BP group performed balance combined with plyometric training three times a week over 6 weeks; while the PL group undertook only plyometric training three times a week during the same period. Meanwhile, both groups were given the same technical training. All participants were tested to assess the COD ability before and after the training period: Southeast Missouri (SEMO) test and 5-0-5 test, dynamic balance ability (Y-Balance test, YBT), and reactive strength index (RSI). Repeated-measure ANOVA revealed that after the intervention there was a significant time $\times$ group interaction for 5-0-5 COD test, YBT of both legs and RSI $\left(p<0.05\right.$, partial $\left.\eta^{2}=0.26-0.58\right)$ due to the better performance observed at post-test compared with a pre-test for the BP group [effect size (ES) = 1.20-1.76], and the improvement was higher than that of the PL group. The change in SEMO test did not differ between BP and PL $\left(\rho<0.159\right.$, partial $\left.\eta^{2}=0.137\right)$, but the magnitude of the with-group improvement for BP $(E S=1.55)$ was higher than that of $P L(E S=0.81)$. These findings suggest that combined training could further improve the COD performance of badminton athletes than plyometric training alone and might provide fitness trainers a more efficient COD training alternative.

Keywords: balance training, plyometrics, change of direction, badminton athletes, badminton

\section{INTRODUCTION}

Badminton is one of the fastest racket sports in the world and is highly competitive and dynamic (Phomsoupha and Laffaye, 2015). During the match, the players perform 6-12 strokes within a rally duration ranging from 6 to $10 \mathrm{~s}$. Due to the fast speed of the shuttlecock and high hitting frequency, the sport exerts a great demand on the abilities of player to run, accelerate, decelerate, jump, lunge, and change direction (Laffaye et al., 2015; Lee and Loh, 2019). A previous study showed that among all physical capacities, the COD performance served as the best physical predictor of badminton excellence $(r=0.74)$ (Hughes and Cosgrove, 2006) so that badminton players maximize such ability 
to enhance on-court success. Until now, several tests for COD ability have been widely used for badminton assessment, such as the Hexagon test, the 5-0-5 COD test, and the Modified SEMO test, and they were verified as representative methods to determine on-court performance of players (Jeyaraman et al., 2012; Ozmen and Aydogmus, 2016; Wong et al., 2019).

In terms of training programs, traditional resistance training has been used to improve the power and COD ability (Brughelli et al., 2008). However, recent studies have revealed that plyometric training and combined plyometric and resistance training presented greater efficiency in improving these abilities (Asadi et al., 2016; Fischetti et al., 2018, 2019). The actual mechanism of plyometric exercise is a lengthening (eccentric contraction) of the muscle-tendon unit followed directly by a shortening or concentric contraction, otherwise termed as a stretch-shortening cycle (SSC) (Markovic and Mikulic, 2010). In practice, it consists of exercises related to jumping, hopping, and skipping with multi-joint actions on stable or unstable surfaces (Negra et al., 2017a,b). While numerous studies have proved that plyometric training could be applied to improve the COD ability in players in racket sports like tennis (Salonikidis and Zafeiridis, 2008; Barber-Westin et al., 2010; FernandezFernandez et al., 2016, 2018), few attempts have been made for badminton (Lim Joe et al., 2012; Majeed and Latheef, 2016; Middleton et al., 2016). Nonnato and colleagues have recently found that plyometric training cannot improve the 5-0-5 COD test performance of professional female soccer players due to a merely small effect on the COD with $180^{\circ}$ angle (Nonnato et al., 2020), and such movement is frequently involved in a badminton match. Therefore, the effect of plyometric training on the COD ability of badminton players remains to be unveiled.

The previous finding has evidenced the importance of balance for the COD performance in that it helps players control the center of gravity (COG) during the accelerations and decelerations phase (Rouissi et al., 2018). For badminton players, balance also plays an important role in addressing issues of controlled COG and other situations challenging their balance, such as twisting movements (particularly of the pivot foot) during jump smash and offensive and defensive attacks. It is logical to undertake balance training to improve movement performance in badminton, particularly COD performance. Previous studies have explored the effect of combined plyometric and balance training on professional female basketball athletes and young soccer players (Makhlouf et al., 2018; Muehlbauer et al., 2019; Bouteraa et al., 2020). The results showed that such a combined program could produce greater performance improvements in balance, power, and COD, as opposed to the single plyometric intervention. However, it was suggested that immaturity or a lack of optimal balance capabilities might compromise the plyometric training adaptations (Bouteraa et al., 2020).

Despite a growing body of literature on combined training in other sport modalities and athletes, there is a paucity of research related to the applications of such training method in badminton. Therefore, it is unclear whether combined training could lead to greater improvement of COD ability in badminton players, or to mention the generalizability of results and practical recommendations from other studies. In order to explore refined fitness programing, the current study was aimed to investigate the effect of 6-week combined balance training and plyometric training on COD performance in elite badminton athletes. We hypothesized that the combined training protocol would further increase the COD and balance performance in badminton athletes when compared with plyometric training alone.

\section{MATERIALS AND METHODS}

\section{Participants}

Sixteen male elite badminton players (eight players had played the quarterfinalists of national youth games and the rest had played the finals at the provincial level) were recruited in this study. The participants belonged to the same club and were physically healthy, free from severe lower-body injuries related to anterior cruciate ligament (ACL), hamstring, meniscus, and ankle, or any medical and orthopedic problems. All players were right-handed and undertook three training sessions per week with each session formed by $2-3 \mathrm{~h}$ of technical and physical training drills (See Figure 1 for participante recruitment process). All participating players volunteered for random allocation to either balance and plyometric training group (BP, $n=8$ ) (age: $20.5 \pm 1.1$ years, height: $177.8 \pm 5.1 \mathrm{~cm}$, weight: $68.1 \pm 7.2 \mathrm{~kg}$, and training experience: $11.4 \pm 1.4$ years) or a control group (PL) (age: $19.1 \pm 2.2$ years, height: $179.1 \pm 6.1 \mathrm{~cm}$, weight: $69.88 \pm 8.94 \mathrm{~kg}$, and training experience: $10.6 \pm 1.1$ years) that performed only plyometric training regimen $(n=8)$. There were no statistically significant differences between the groups in these personal characteristics. Before data collection, the participants were informed about the benefits and possible risks associated with the study, and the participants provided written informed consent to participate. The players were in their normal routine of diet and had caffeine-free beverages during the whole study period. The study protocol was approved by the Beijing Sport University Institutional Research Commission (Approval number: $2020008 \mathrm{H}$ ), and all procedures were conducted in accordance with the Declaration of Helsinki.

\section{Procedures}

All experimental training programs were conducted along with a weekly technical training routine. Participants from the BP and PL groups followed a balance combined with a plyometric training program (40 min of plyometrics and $20 \mathrm{~min}$ of balance training) three times per week with 24$48 \mathrm{~h}$ of recovery between each training session. In order to control the 20-min balance training protocol, the PL group was required to perform the same drills as the BP group. However, unlike the latter that undertook all the exercises under unstable conditions (i.e., BOSU ball, Swiss ball, and Balance pad), they practiced on the floor. Before the commencement of the study and the initiation of testing, all players completed a 2-week trial period (three sessions/week) in order to become familiarized with the physical training programs during the formal experimental course of the study. A detailed description of balance and plyometric training protocols and biweekly progression are presented in Tables 1, 2. During each session, 


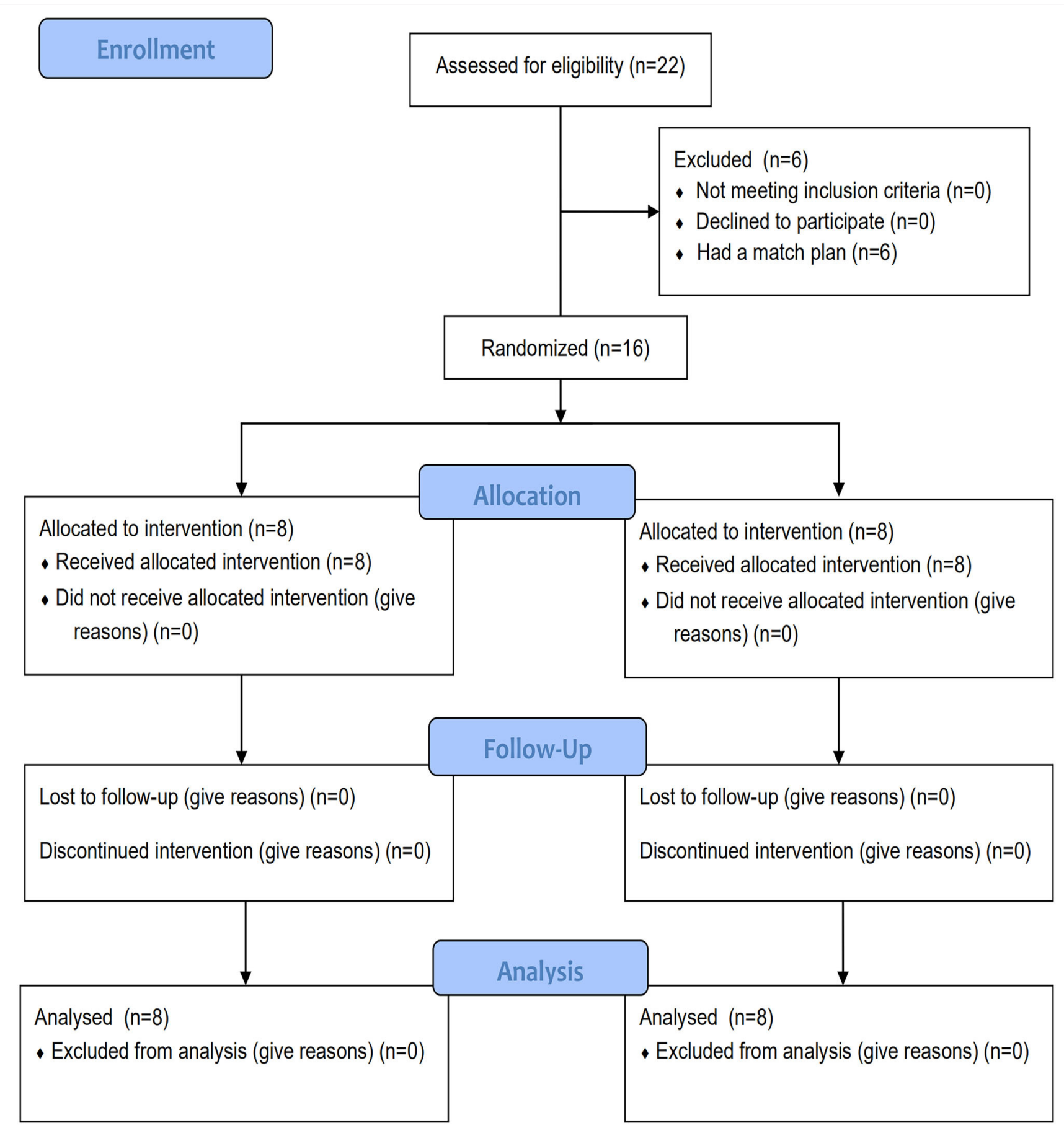

FIGURE 1 | Flow diagram of the participants recruitment process.

players received consistent instructions from certified strength and conditioning coaches on proper techniques for agility drills, balance exercises, plyometric exercises, and landing. All the protocols were designed and supervised by one of the authors, who is an experienced researcher in strength and conditioning, and a fitness trainer with a master degree in strength and conditioning, who works as the fitness coach for the Chinese
National Junior Team (U-17) of Badminton and collegiate team of badminton and has been certified by the State General Administration of Sport.

\section{Test Program}

Data of COD testing were collected before and after the implementation of the 6-week training intervention at the indoor 
TABLE 1 | The balance training program for balance-plyometric (BP) (combined training) group.

\begin{tabular}{|c|c|c|c|}
\hline Exercises & The first stage (1-2 weeks) & The second stage (3-4 weeks) & The third stage (5-6 weeks) \\
\hline Stand on the balance board exercise & $\begin{array}{l}\text { Static standing on the board with } \\
\text { two legs } \\
\text { (3 sets: } 30 \text { s/set) }\end{array}$ & $\begin{array}{l}\text { Static standing on the board with } \\
\text { two legs and eyes closed } \\
\text { (3 sets: } 30 \text { s/set) }\end{array}$ & $\begin{array}{l}\text { Squat on the plate with eyes } \\
\text { closed } \\
\text { (3 sets: } 10 \text { reps/set) }\end{array}$ \\
\hline Supine straight leg bridge on Swiss Ball & $\begin{array}{l}\text { Isometric supine straight leg } \\
\text { bridge on Swiss Ball } \\
\text { (3 sets: } 30 \text { s/set) }\end{array}$ & $\begin{array}{l}\text { Isometric supine single-leg } \\
\text { bending bridge on Swiss Ball } \\
\text { (3 sets: } 30 \text { s/set) }\end{array}$ & $\begin{array}{l}\text { Dynamic supine single-leg } \\
\text { bending bridge on Swiss } \\
\text { (3 sets: } 10 \text { reps/set) }\end{array}$ \\
\hline Side-plank with inflated balance disc & $\begin{array}{l}\text { Side-plank with inflated balance } \\
\text { disc with elbow } \\
\text { (3 sets: } 30 \text { s/set) }\end{array}$ & $\begin{array}{l}\text { Side-plank with inflated balance } \\
\text { disc and the non-supporting leg } \\
\text { stretches backward } \\
\text { (3 sets: } 10 \text { reps/set) }\end{array}$ & $\begin{array}{l}\text { Side-plank with inflated balance } \\
\text { disc and the non-supporting leg } \\
\text { stretches backward with elastic } \\
\text { band } \\
\text { (3 sets: } 10 \text { reps/set) }\end{array}$ \\
\hline Lunge squat on BOSU ball & $\begin{array}{l}\text { Lunge squat on BOSU ball } \\
\text { (3 sets: } 10 \mathrm{reps} / \mathrm{leg} / \mathrm{set})\end{array}$ & $\begin{array}{l}\text { Lunge squat on BOSU ball and } \\
\text { inflated balance disc } \\
\text { (3 sets: } 10 \text { reps/leg/set) }\end{array}$ & $\begin{array}{l}\text { Lunge squat on BOSU ball and } \\
\text { inflated balance disc with } 5 \mathrm{~kg} \\
\text { dumbbells } \\
\text { ( } 3 \text { sets: } 10 \text { reps/leg/set) }\end{array}$ \\
\hline Airex ${ }^{\circledR}$ Balance-pad Elite exercise & $\begin{array}{l}\text { Single-leg squat with } \\
\text { balance-pad } \\
\text { (3 sets: } 10 \text { reps/leg/set) }\end{array}$ & $\begin{array}{l}\text { Single-leg standing with } \\
\text { balance-pad and the } \\
\text { non-supporting leg stretches } \\
\text { backward } \\
\text { (3 sets: } 12 \text { reps/leg/sets) }\end{array}$ & $\begin{array}{l}\text { Single-leg support with } \\
\text { balance-pad elite and the } \\
\text { non-supporting leg stretches } \\
\text { backward with elastic band } \\
\text { (3 sets: } 12 \text { reps/leg/sets) }\end{array}$ \\
\hline Rest & & een exercise: $60 \mathrm{~s}$ Between sets: 3 min & \\
\hline
\end{tabular}

TABLE 2 | The plyometric training program for BP and plyometric $(\mathrm{PL})$ training group.

\begin{tabular}{|c|c|c|c|}
\hline Exercises & The first stage (1-2 weeks) & The second stage ( $3-4$ weeks) & The third stage (5-6 weeks) \\
\hline Front barrier jump (6 hurdles) & $\begin{array}{l}\text { Double-leg front barrier jump } \\
(15 \mathrm{~cm}) \\
\text { (3 sets: } 10 \text { reps/set) }\end{array}$ & $\begin{array}{l}\text { Single-leg front barrier jump } \\
(15 \mathrm{~cm}) \\
\text { (3 sets: } 5 \text { reps/leg/set) }\end{array}$ & $\begin{array}{l}\text { Single-leg front barrier jump } \\
(30 \mathrm{~cm}) \\
\text { (4 sets: } 5 \text { reps/leg/set) }\end{array}$ \\
\hline Lateral high-knees with hurdles & $\begin{array}{l}\text { 4-hurdle }(15 \mathrm{~cm}) \\
\text { (3 sets: } 2 \text { reps/set) }\end{array}$ & $\begin{array}{l}\text { 6-hurdle }(30 \mathrm{~cm}) \\
\text { (3 sets: } 4 \text { reps/set) }\end{array}$ & $\begin{array}{l}\text { 6-hurdle }(30 \mathrm{~cm}) \\
\text { (3 sets: } 6 \text { reps/set) }\end{array}$ \\
\hline Lateral barrier jump & $\begin{array}{l}\text { Double-leg jump (15 cm) } \\
\text { (3 sets: } 10 \text { reps/set) }\end{array}$ & $\begin{array}{l}\text { Double-leg jump (30 cm) } \\
\text { (3 sets: } 12 \text { reps/set) }\end{array}$ & $\begin{array}{l}\text { Single-leg jump (30 cm) } \\
\text { (3 sets: } 15 \text { reps/leg/set) }\end{array}$ \\
\hline Depth jump & $\begin{array}{l}\text { Jump with } 20 \mathrm{~cm} \text { box } \\
\text { (3 sets: } 8 \text { reps/set) }\end{array}$ & $\begin{array}{l}\text { Jump with } 30 \mathrm{~cm} \text { box } \\
\text { (3 sets: } 8 \text { reps/set) }\end{array}$ & $\begin{array}{l}\text { Jump with } 40 \mathrm{~cm} \text { box } \\
\text { (3 sets: } 8 \text { reps/set) }\end{array}$ \\
\hline Multi-direction jumps with hurdles & $\begin{array}{l}\text { Triangle jump with double-leg } \\
\text { ( } 3 \text { hurdles) } \\
\text { ( } 3 \text { sets: } 6{ }^{\star} 3 \text { reps/set) }\end{array}$ & $\begin{array}{l}\text { Square jump with single-leg } \\
\text { ( } 4 \text { hurdles) } \\
\text { ( } 3 \text { sets: } 8^{\star} 3 \text { reps/set) }\end{array}$ & $\begin{array}{l}\text { Hexagon jump with single-leg } \\
\text { ( } 6 \text { hurdles) } \\
\text { ( } 3 \text { sets: } 12^{\star} 3 \text { reps/set) }\end{array}$ \\
\hline Intensity and number of contact with ground & $\begin{array}{l}\text { Low intensity } \\
144\end{array}$ & $\begin{array}{l}\text { Middle intensity } \\
234\end{array}$ & $\begin{array}{l}\text { High intensity } \\
325\end{array}$ \\
\hline Rest & \multicolumn{3}{|c|}{ Between exercise: $60 \mathrm{~s}$ Between sets: $3 \mathrm{~min}$} \\
\hline
\end{tabular}

sports science center and badminton court of local institution of authors. The testing consisted of Modified SEMO Test, Modified 5-0-5 COD test, Y-balance test (YBT), and reactive strength index (RSI) test, and they were performed and completed within 1 day. Players were already accustomed to the testing procedures used in this research as they routinely performed these tests in the club. Prior to testing, participants completed a warmup that included a 5-min dynamic stretching, 8-min movement integration, and 2-min neural activation. Post-training testing was performed 3 days after the last training session to ensure optimal recovery, and no intensive training was implemented $24 \mathrm{~h}$ before testing to prevent the effects of fatigue. Each testing was completed at the same time of the day, at the same site, with the same sporting shoes, and surveilled by the same investigators. The set of tests and measurements were detailed in the following context:

\section{Modified SEMO Test}

This test evaluates the capacity of making quick changes of direction, forward sprints, diagonal backpedaling, and side shuffling, which are the most frequent movements performed during badminton matches (Jeyaraman et al., 2012). The test area was set up on the half of the badminton court with a length of $6.70 \mathrm{~m}$ and a width of $6.10 \mathrm{~m}$. Four cones were placed on four corners of the court to mark each site of change of direction (see Figure 2). At each trial, participants first started 
side shuffling (facing the court) on instructions of the coach, and then back-pedal diagonally across the court, sprint forward, back-pedal diagonally across court again, sprint forward, and finally side-shuffle (facing the court) to the finishing point. Test times were recorded using SmartSpeed PT Timing Gate System (Fusion Sport, Coopers Plains, Australia), which was installed at the start/finish point of the test. Three consecutive trials were performed and separated by $2 \mathrm{~min}$ of passive rest, with the highest values being recorded for analysis (intraclass correlation coefficients of a two-way random model: $0.85,95 \%$ CI: $[0.74,0.92])$.

\section{Modified 5-0-5 COD Test}

The 5-0-5 COD test is widely used to evaluate the COD ability of athletes in various sports, which is very suitable for those sports that require short-distance acceleration and COD, such as basketball, rugby, tennis, and badminton (Hughes and Bopf, 2005). Due to the short moving distance in the badminton court, players often involve in COD with $180^{\circ}$ angle, which is highly similar to the 5-0-5 COD test. In order to suit badminton specifically, the researcher adjusted the running distance of the test to be similar to the distance inside the badminton court, as shown in Figure 3. Two cone buckets A were placed symmetrically at the starting line, then two other cone buckets $\mathrm{B}$ were placed at a position $3 \mathrm{~m}$ to the right of the parallel $\mathrm{A}$ point, and finally, the cone bucket $\mathrm{C}$ was placed at a position $3 \mathrm{~m}$ to the left of the parallel A point. Smart Speed (Fusion Sport, Coopers Plains, Australia) was placed behind each pair of cones. After hearing the command "Ready, start," the participants made a quick right turn and ran from A to B. Upon arrival, the participant quickly turned around and sprinted to C, then quickly turned around and ran back to A. Each participant performed three tests, and the best of the three tests was the final valid score. There was a 5-10 min recovery period between each test. Smart Speed was automatically timed at the beginning and the end of the test. Three consecutive trials were performed and separated by $2 \mathrm{~min}$ of passive rest, with the highest values being recorded for analysis (intraclass correlation coefficients: 0.88 [0.79, 0.94]).

\section{Y-Balance Test}

The test was used to assess the dynamic balance of players (Shaffer et al., 2013). While barefoot, participants balanced themselves with one foot on the center board of a commercially available YBT instrument (Move2Perform, Evansville, IN). To perform the test, they need to place their hands on the hips and reach as far as possible by pushing the board with the reaching limb into the anterior, posteromedial, and posterolateral directions and return to the original start position (see Figure 4 for the illustration). Reach distance was measured at the nearest edge of the reach indicator to the closest $0.5 \mathrm{~cm}$. All participants performed three practice trials in each of three directions on each leg before three formals test trials. YBT reach distances were normalized to leg length (\%), which was obtained by measuring the length from the right anterior superior iliac spine of the players to right medial malleolus in supine. The highest composite score was recorded

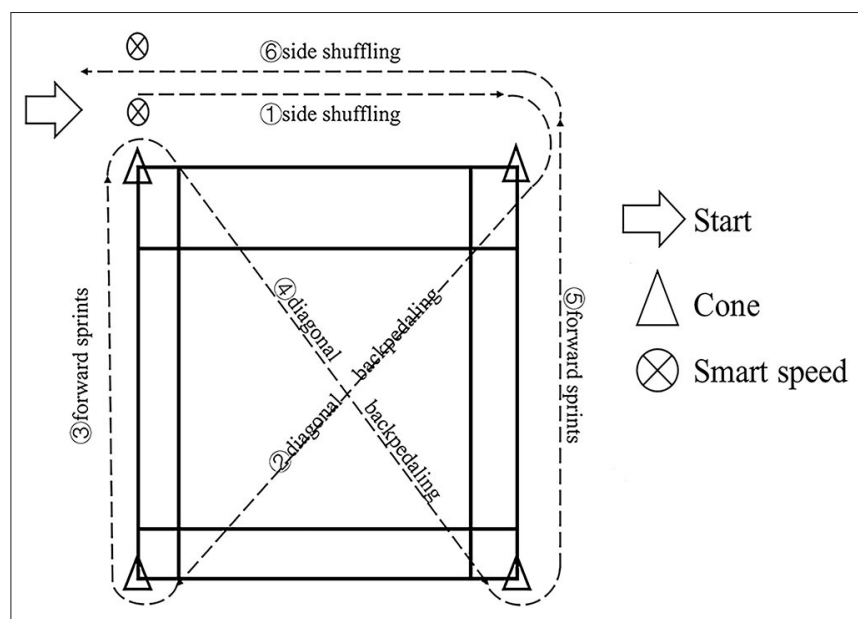

FIGURE 2 | Modified Southeast Missouri (SEMO) test.

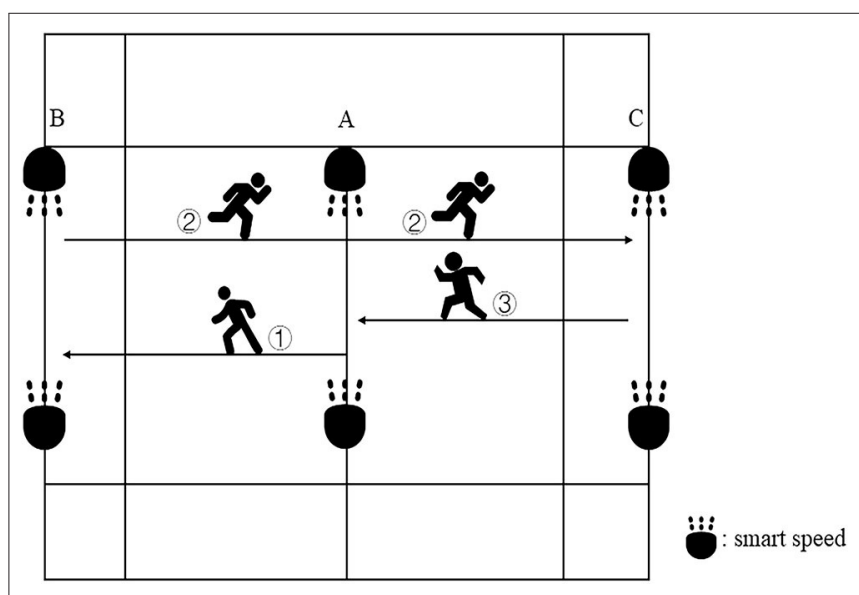

FIGURE 3 | Modified 5-0-5 COD test.

for analysis using the following formula:

$$
\begin{aligned}
& \text { Composite Score } \\
& =\frac{(\text { Anterior }+ \text { Posteromedial }+ \text { Posterolateral })}{3 \times \text { Right Limb Length }} \times 100
\end{aligned}
$$

where anterior, posteromedial and posterolateral represent the distances reached in each direction (intraclass correlation coefficients: $0.92[0.85,0.96])$.

\section{Reactive Strength Index Test}

The test is applied to evaluate how athletes perform during plyometrics by measuring the muscle-tendon stress and their reactive jump capacity so that their ability to quickly and effectively moving through the strength-shortening cycle is demonstrated (Ebben and Petushek, 2010). In practice, the RSI is strongly related to COD speed and acceleration speed and depth jump from the height of $30 \mathrm{~cm}$ could provide a valid result of RSI (Flanagan et al., 2008; Byrne et al., 2017). Therefore, in this study, the RSI was measured using depth jump from a $30 \mathrm{~cm}$ plyometric 

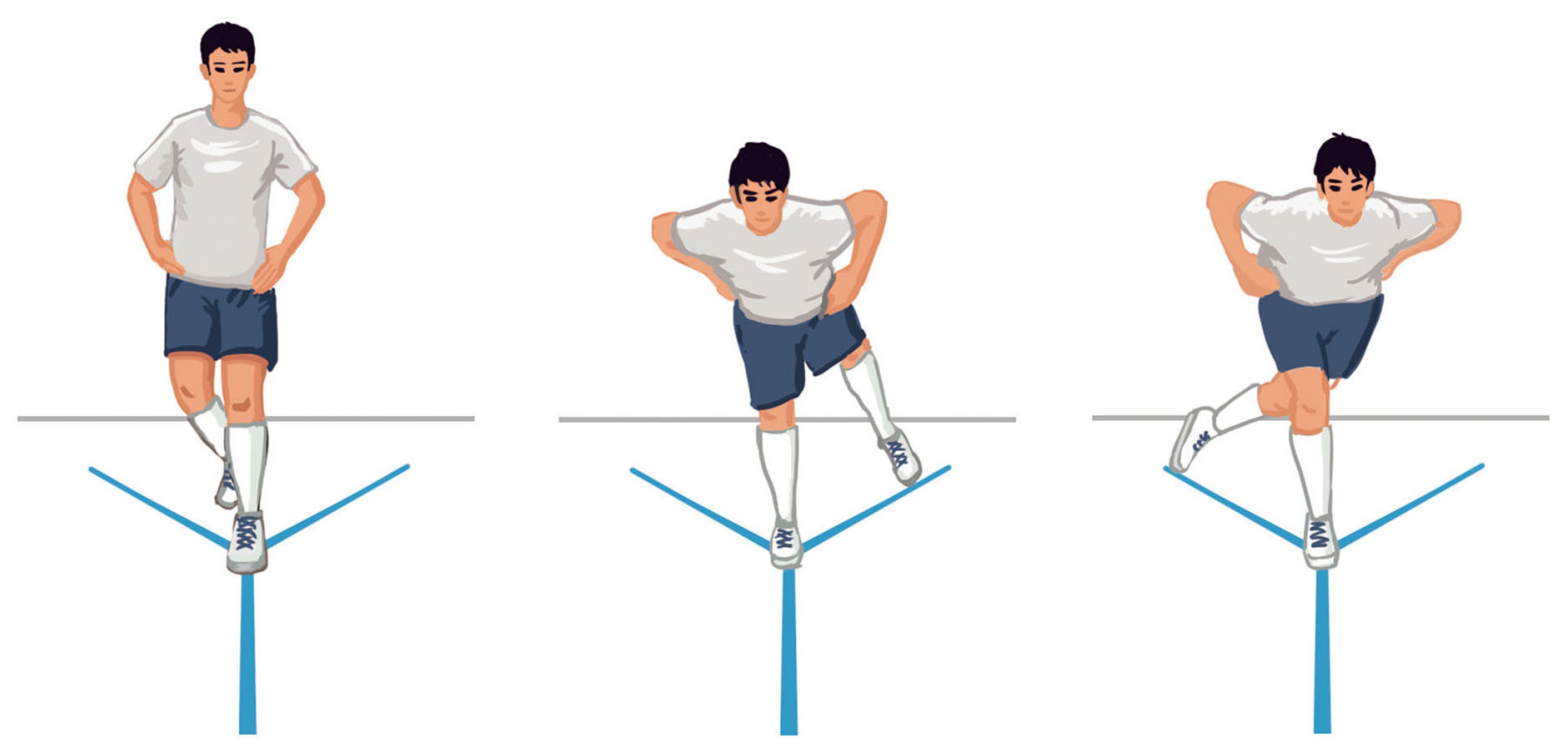

FIGURE 4 | Y-balance test (YBT).

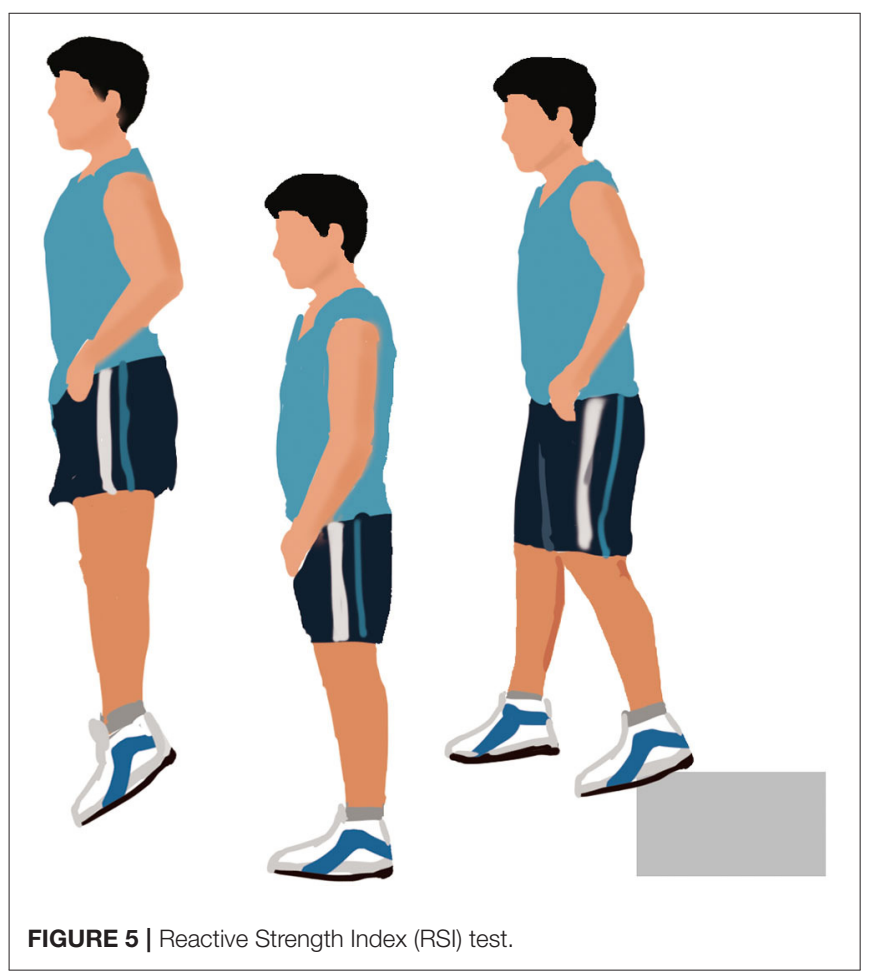

box (see Figure 5). During the test, participants were instructed to perform the depth jump with two hands on their hips and step forward off the box without stepping down or jumping up. After observing the demonstration, each player was then allowed to carry out two practice trials before formal testing. On falling, players would land on an in-ground force plate (Kistler 9281CA, Winterthur, Switzerland) that has a sampling frequency of $1,000 \mathrm{~Hz}$. They were requested to jump as high and quickly as possible after landing, and the jump height was calculated from take-off velocity derived from their respective force-time data. Later, the RSI score was calculated using the highest jump height recorded from three trials using the below formula (intraclass correlation coefficients: $0.86[0.75,0.93])$ :

$$
\mathrm{RSI}=\frac{\text { Jump height }}{\text { ground contact time }}
$$

\section{Statistical Analysis}

Data are presented as means and SD. The normality of data distribution was confirmed using the Shapiro-Wilk test. Training-related effects were assessed by 2-way repeatedmeasure ANOVA (group $\times$ time) on SEMO Test, 5-0-5 COD test, YBT, and RSI, with the Greenhouse-Geisser adjustment was applied. Partial $\eta^{2}$ was used as the effect size (ES) estimation for the time by group interaction effect with its strength being interpreted as the following: $<0.06$ as small, $<0.14$ as moderate, and $\geq 0.14$ as large (Cohen, 1988), while the Cohen's $d$ converted from partial $\eta^{2}$ was used to represent the ES of main effect (Cohen, 1988). When a significant effect was found, Bonferonni post-hoc correction was performed to identify pairwise differences. The absolute value of each test result was used to calculate the ES for the within- and betweengroup comparisons, represented as Cohen's $d$. It was interpreted according to the following thresholds: $<0.2$ as trivial, $0.2-0.6$ as small, $0.6-1.2$ as moderate, $1.2-2.0$ as large, and $>2.0$ as very large (Hopkins et al., 2009). The Statistical Package for Social Sciences (SPSS Inc., Chicago, IL, USA, version 22.0) was used for all analyses. The level of significance was set at $p<0.05$ for all tests.

\section{RESULTS}

Table 3 presents the descriptive statistics of all COD tests, results of repeated ANOVA for pre-and post-training fitness testing, 
TABLE 3 | Descriptive statistics of agility test results for BP group and PL group before and after the 6-week training intervention.

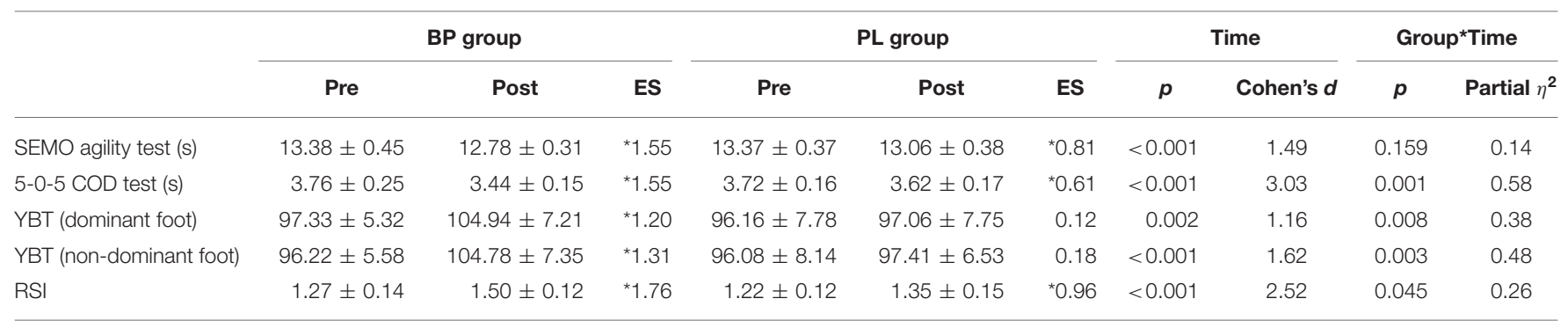

*Statistically significant difference between pre- and post-test, $p<0.05$.

SEMO, modified southeast Missouri; 5-0-5 COD test = 5-0-5 change of direction test; YBT, Y-Balance test; RSI, reactive strength index.

and corresponding ESs. No statistically significant differences between groups were found at baseline for all test measures.

For both BP and PL groups, training resulted in statistically significant improvement in SEMO test, 5-0-5 COD test, and RSI $\left(p<0.001\right.$, partial $\eta^{2}=0.598,0.835$, and 0.783 , respectively), but BP showed higher ESs than PL (1.55 vs. 0.81, 1.55 vs. 0.61 , and 1.76 vs. 0.96 ). Significant group by time interaction effects were shown between groups for 5-0-5 COD test $\left[F_{(1,14)}=19.273, p=0.001\right.$, and partial $\left.\eta^{2}=0.579\right]$, indicating a significantly greater improvement in the above test performance after the BP intervention when compared with the PL intervention. Moreover, a statistically significant increase in YBT was shown for the BP group on both dominant and non-dominant feet $(E S=1.20$ and 1.31 ). Significant group by time interaction effects were shown between groups for YBT (dominant foot) $\left[F_{(1,14)}=8.710, p=0.008\right.$, and partial $\eta^{2}$ $=0.384]$, YBT (non-dominant foot) $\left[F_{(1,14)}=12.674, p=\right.$ 0.003 , and partial $\left.\eta^{2}=0.475\right]$ and RSI $\left[F_{(1,14)}=4.831, p\right.$ $=0.045$, and partial $\eta^{2}=0.257$, indicating a significantly greater improvement in the above test performance after the BP intervention when compared with the PL intervention. Figure 6 depicts within- and between-group ESs for comparisons of test results.

\section{DISCUSSION}

The purpose of the study was to investigate the effectiveness of the 6-week combined balance and plyometric training program on the COD performance of elite badminton players. To the best of our knowledge, this is the first study comparing the effect between combined training and plyometric training. Based on the results, we found the combined protocol to be more effective in improving COD ability, dynamic balance, and RSI of players when compared with the latter. As COD ability, dynamic balance, and RSI are valid indicators of the movement performance of badminton players (Hughes and Bopf, 2005; Masu et al., 2014), the current findings could help enrich the badmintonspecific strength and conditioning routines and improve the on-court performance.

Findings of the study imply that 6 weeks of BP traininginduced better adaptations in COD, balance, and reactive strength performances. As the well-developed COD ability requires not only strong lower limb power to move quickly, but also a good balance to control body posture and to overcome the inertia caused by decelerations and brakings, adding an extra balance training indeed played an important role in boosting such performance (Sekulic et al., 2013). Former research documented a high correlation $(r=0.83)$ of COD ability with badminton performance during competitive matches (Tiwari et al., 2011), which are characterized by high-intensity rallies with short rest intervals (Alam et al., 2010). Although the directions and trajectories of shuttlecock are fixed during rallies, they are randomly determined by players at milliseconds of time before each stroke (Lees, 2003). Therefore, players are requested to execute rapid CODs, consecutive jumps, lunges, and multiple accelerations and decelerations (Manrique and Gonzalez-Badillo, 2003), and possessing decent COD is the key to applying the most efficient footwork into reaching the correct places and hitting the shuttlecock. As previously revealed that the most frequent moving patterns during badminton matches were forward-backward movements, lateral movements, and CODs (Phomsoupha and Laffaye, 2015), results of the SEMO Test and the 5-0-5 COD test would be a performance indicator of on-court COD of a player. Meanwhile, although sufficient investigations have already evidenced positive adaptations in the COD ability of athletes after plyometric training (Manouras et al., 2016; Asadi et al., 2017), the current study provided novel findings that a combined training could induce greater improvement, which is in consensus with findings of Bouteraa et al. (2020) when applying similar training methodology to female basketball players.

The results further revealed a significant increase in both YBalance and RSI performance for the BP group, while reporting only meaningful adaptation in RSI for the PL group. It could be inferred from this result that badminton-specific COD ability has been improved after plyometric training, but not as much as a combined training protocol, which is consistent with the previous research where significant adaptations were reported in female basketball players and ordinary adolescents after combined training (Chaouachi et al., 2014; Bouteraa et al., 2020). RSI is an indicator of how efficiently athletes perform the SSC. Although plyometric training has been shown to significantly improve the efficiency of SSC utilization (Jeffreys et al., 2019; Dallas et al., 2020), it is interesting to note that the BP group exhibited a greater increase in RSI. A potential reason could be that balance training enhanced the body control of players 

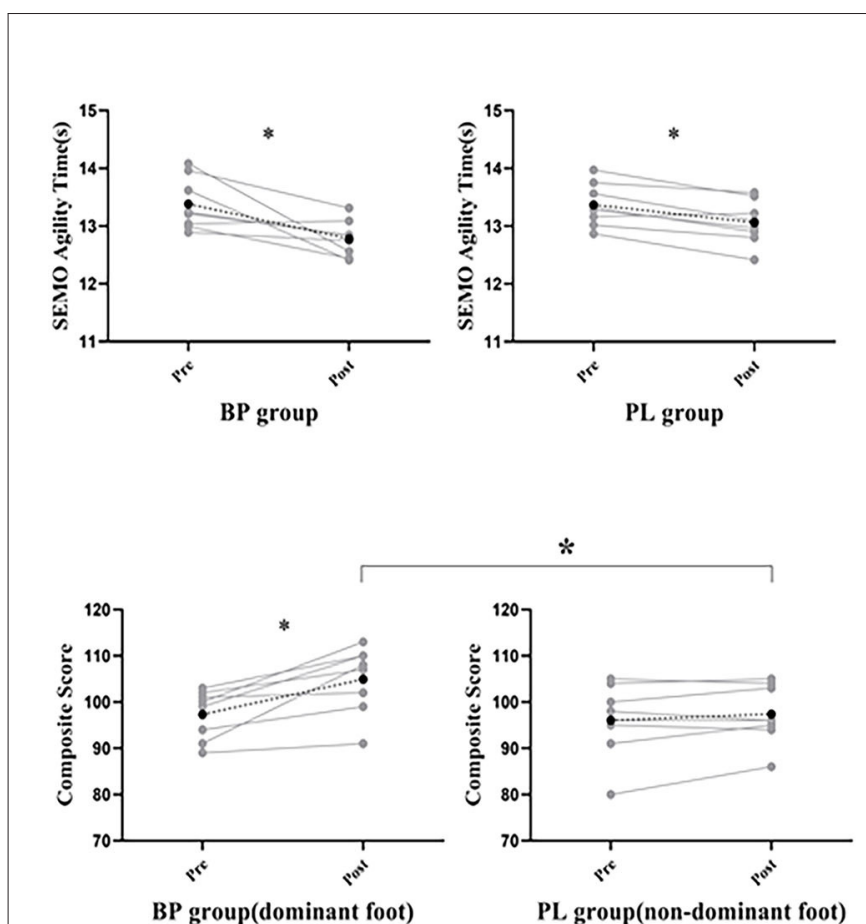

PL group(non-dominant foot)

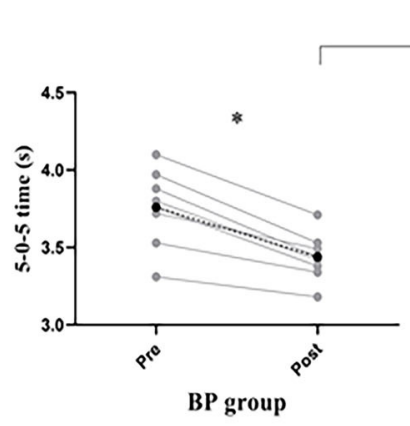

$*$

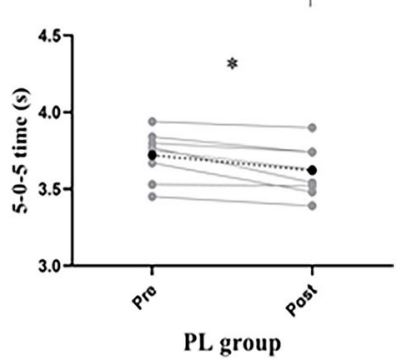

$*$

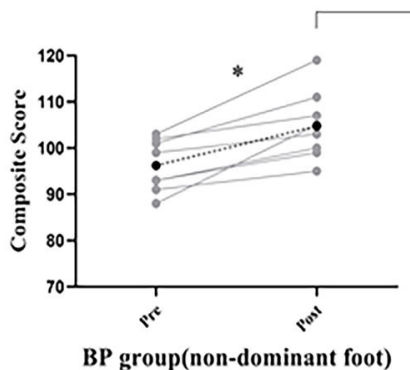

BP group(non-dominant foot)

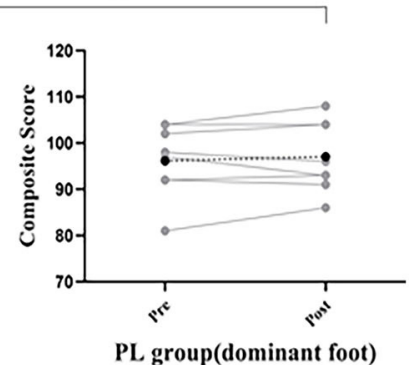

PL group(dominant foot)
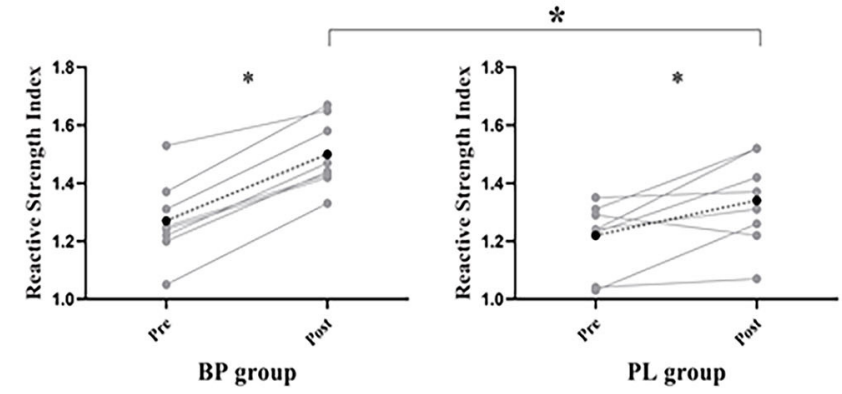

FIGURE 6 | Individual and mean values for agility related tests during before and after Balance-Plyometric $(\mathrm{BP})$ and $\mathrm{Plyometrics}(\mathrm{PL})$ training. Note: ${ }^{*} p<0.05$.

and finally reduced the time spent on the landing during jumps. It is also possible that balance training could be beneficial for the power improvement of the lower limbs. Kean et al. (2006) found a $10 \%$ increase in vertical jumping after 5-week balance training, which helped decrease the sway of the COG, allowing athletes to land more consistently with optimal vertical jump angles. Lower limb reactive strength and posture control are important factors affecting quick COD of athletes, body stability as well as effective prevention of injuries (Borghuis et al., 2008). In this regard, the combined training paradigm could be more preferable for the complex, high-speed, unilateral, repetitive dynamic badminton movements that request a high extent of postural control.

To understand the benefit of combined training to badminton performance from a physiological point of view, the coordinated work of the visual system, vestibular system, and proprioceptive system should be addressed. When a player is changing his directions quickly and the body is imbalanced, the aforementioned systems will work together to regain the balance and maintain body stiffness. During this process, the proprioceptors (muscle spindles and Golgi tendons) and other joint receptors will sense changes in body posture, and then the vestibular system will send feedback to the central nervous system to generate kinesthetic awareness. Subsequently, in order to control body balance, the central nervous system needs to transmit signals to effectors (muscles), which will ultimately respond appropriately by adjusting the COG of the body (Sekulic et al., 2013). Therefore, the ability to change direction and make fast acceleration/deceleration is attributed to the joint effort of proprioceptors and effectors. In essence, the plyometric training could only strengthen the function of the effector (muscle), but not the proprioceptive system, which would be improved via balance training. Therefore, the combined balance and plyometric training should be prioritized in boosting both 
the performance of the proprioceptive system and muscle function simultaneously.

The results of our study evidenced the promising effects of the combined training paradigm on badminton COD ability so that it seems feasible to apply balance training into the already existed plyometric training routines of players. Moreover, from a practical perspective, fitness coaches are suggested to vary the format and sequence of exercises, training volumes, and intensities of such training prescription in order to add more stimuli. For example, instead of separating plyometrics and balance training apart, coaches could adopt a circuit training protocol that involves two training modalities in each circuit, using balance training as an alternative for passive rest between different sets of exercises. Finally, coaches and sports scientists are suggested to investigate whether incorporating reaction training drills to combined training protocol could further improve badminton on-court COD performance.

Despite the new information about the effect of combined training on badminton COD ability, several limitations need to be acknowledged. First, due to the fact that most elite male badminton players usually have fixed match and training planning (traveling to compete and train) that are not possible to be alternated, the study could only include a comparatively small sample size. Second, it should be noted that an experiment period of 6 weeks was chosen in order to verify that a shorter intervention than the commonly-used 8 weeks to induce favorable adaptation, as the experiment schedule had to be acceptable for both the coaches and players. Researchers should be, therefore, cautious when interpreting and generalizing the current findings.

\section{CONCLUSIONS}

In summary, the 6-week training intervention for elite male badminton players showed that combined training (BP) induced an overall better adaptation for the SEMO TEST, the 5-0-5 COD test, the YBT, and the RSI test than plyometric training

\section{REFERENCES}

Alam, F., Chowdhury, H., Theppadungporn, C., Subic, A., and Khan, M. (2010). Aerodynamic properties of badminton shuttlecock. Int. J. Mech. Mater. Eng. 2, 2487-2492. doi: 10.1016/j.proeng.2010.04.020

Asadi, A., Arazi, H., Young, W. B., and de Villarreal, E. S. (2016). The effects of plyometric training on change-of-direction ability: a meta-analysis. Int. J. Sports Physiol. Perform. 11, 563-573. doi: 10.1123/ijspp.2015-0694

Asadi, A., Ramirez-Campillo, R., Meylan, C., Nakamura, F. Y., Cañas-Jamett, R., and Izquierdo, M. (2017). Effects of volume-based overload plyometric training on maximal-intensity exercise adaptations in young basketball players. J. Sports Med. Phys. Fitness 57, 1557-1563. doi: 10.23736/s0022-4707.16.06640-8

Barber-Westin, S. D., Hermeto, A. A., and Noyes, F. R. (2010). A six-week neuromuscular training program for competitive junior tennis players. J. Strength Cond. Res. 24, 2372-2382. doi: 10.1519/JSC.0b013e3181e $8 \mathrm{a} 47 \mathrm{f}$

Borghuis, J., Hof, A. L., and Lemmink, K. A. (2008). The importance of sensory-motor control in providing core stability. Sports Med. 38, 893-916. doi: 10.2165/00007256-200838110-00002
(PL). Moreover, considering the completion of balance training is relatively time-efficient ( $20 \mathrm{~min}$ in the current study), it is suggested that adding such exercises would allow optimal training adaptation for badminton players.

\section{DATA AVAILABILITY STATEMENT}

The raw data supporting the conclusions of this article will be made available by the authors, without undue reservation.

\section{ETHICS STATEMENT}

The studies involving human participants were reviewed and approved by Beijing Sport University Institutional Research Commission (Approval number: 2020008H). The patients/participants provided their written informed consent to participate in this study.

\section{AUTHOR CONTRIBUTIONS}

$\mathrm{ZG}, \mathrm{YH}$, and DB: conceptualization. ZG, YH, YC, and DB: methodology, validation, and writing-original draft preparation. ZG and YC: software and visualization. ZG, WG, and YC: formal analysis. ZG, ZZ, and BL: investigation. BL and DB: resources. ZG and $\mathrm{YH}$ : data curation. $\mathrm{ZG}, \mathrm{YH}$, and $\mathrm{YC}$ : writing-review and editing. DB and YC: supervision, project administration, and funding acquisition. All authors have read and agreed to the published version of the manuscript.

\section{FUNDING}

This work was supported in part by the National Key Research and Development Program of China under Grant 2020AAA0103404, the National Natural Science Foundation of China under Grant 72071018, and the Fundamental Research Funds for the Central Universities under Grant 2021TD008.

Bouteraa, I., Negra, Y., Shephard, R. J., and Chelly, M. S. (2020). Effects of combined balance and plyometric training on athletic performance in female basketball players. J. Strength Cond. Res. 34, 1967-1973. doi: 10.1519/JSC.0000000000002546

Brughelli, M., Cronin, J., Levin, G., and Chaouachi, A. (2008). Understanding change of direction ability in sport: a review of resistance training studies. Sports Med. 38, 1045-1063. doi: 10.2165/00007256-200838120-00007

Byrne, D. J., Browne, D. T., Byrne, P. J., and Richardson, N. (2017). Interday reliability of the reactive strength index and optimal drop height. J. Strength Condition. Res. 31, 721-726. doi: 10.1519/JSC.0000000000001534

Chaouachi, A., Othman, A. B., Hammami, R., Drinkwater, E. J., and Behm, D. G. (2014). The combination of plyometric and balance training improves sprint and shuttle run performances more often than plyometriconly training with children. J. Strength Condition. Res. 28, 401-412. doi: 10.1519/JSC.0b013e3182987059

Cohen, J. (1988). Statistical Power Analysis for the Behavioral Sciences, 2nd Edn. Hillsdale, NJ: Lawrence Earlbaum Associates.

Dallas, G. C., Pappas, P., Ntallas, C. G., Paradisis, G. P., and Exell, T. A. (2020). The effect of four weeks of plyometric training on reactive strength index 
and leg stiffness is sport dependent. J. Sports Med. Phys. Fitness 60, 979-984. doi: 10.23736/S0022-4707.20.10384-0

Ebben, W. P., and Petushek, E. J. (2010). Using the reactive strength index modified to evaluate plyometric performance. J. Strength Condition. Res. 24, 1983-1987. doi: 10.1519/JSC.0b013e3181e72466

Fernandez-Fernandez, J., De Villarreal, E. S., Sanz-Rivas, D., and Moya, M. (2016). The effects of 8-week plyometric training on physical performance in young tennis players. Pediatric Exerc. Sci. 28, 77-86. doi: 10.1123/pes.2015-0019

Fernandez-Fernandez, J., Granacher, U., Sanz-Rivas, D., Marín, J. M. S., Hernandez-Davo, J. L., and Moya, M. (2018). Sequencing effects of neuromuscular training on physical fitness in youth elite tennis players. $J$. Strength Condition. Res. 32, 849-856. doi: 10.1519/JSC.0000000000002319

Fischetti, F., Cataldi, S., and Greco, G. (2019). A combined plyometric and resistance training program improves fitness performance in 12 to 14-years-old boys. Sport Sci. Health 15, 615-621. doi: 10.1007/s11332-019-00560-2

Fischetti, F., Vilardi, A., Cataldi, S., and Greco, G. (2018). Effects of plyometric training program on speed and explosive strength of lower limbs in young athletes. J. Phys. Educ. Sport 18, 2476-2482. doi: 10.7752/jpes.2018.04372

Flanagan, E. P., Ebben, W. P., and Jensen, R. L. (2008). Reliability of the reactive strength index and time to stabilization during depth jumps. J. Strength Condition. Res. 22, 1677-1682. doi: 10.1519/JSC.0b013e318182034b

Hopkins, W., Marshall, S., Batterham, A., and Hanin, J. (2009). Progressive statistics for studies in sports medicine and exercise science. Med. Sci. Sports Exerc. 41, 3-13. doi: 10.1249/MSS.0b013e31818cb278

Hughes, M., and Bopf, G. (2005). Relationships between performance in jump tests and speed tests in elite badminton players. J. Sports Sci. 23, 194-195.

Hughes, M., and Cosgrove, M. (2006). “Assessment of elite badminton athletes”, in Sport and Exercise Physiology Testing Guidelines, the British Association of Sport and Exercise Sciences Guide. Routledge. 1, 214-219.

Jeffreys, M. A., De Ste Croix, M. B. A., Lloyd, R. S., Oliver, J. L., and Hughes, J. D. (2019). The effect of varying plyometric volume on stretch-shortening cycle capability in collegiate male rugby players. J. Strength Cond. Res. 33, 139-145. doi: 10.1519/JSC.0000000000001907

Jeyaraman, R., District, E., and Nadu, T. (2012). Prediction of playing ability in badminton from selected anthropometrical physical and physiological characteristics among inter collegiate players. Int. J. Adv. Innov. Res. 2:11.

Kean, C. O., Behm, D. G., and Young, W. B. (2006). Fixed foot balance training increases rectus femoris activation during landing and jump height in recreationally active women. J. Sports Sci. Med. 5, 138-148.

Laffaye, G., Phomsoupha, M., and Dor, F. (2015). Changes in the game characteristics of a badminton match: a longitudinal study through the olympic game finals analysis in men's singles. J. Sports Sci. Med. 14, 584-590.

Lee, J. J. J., and Loh, W. P. (2019). A state-of-the-art review on badminton lunge attributes. Comput. Biol. Med. 108, 213-222. doi: 10.1016/j.compbiomed.2019.04.003

Lees, A. (2003). Science and the major racket sports: a review. J. Sports Sci. 21, 707-732. doi: 10.1080/0264041031000140275

Lim Joe, H., Wee Eng, H., Chan Kai, Q., and Ler Hui, Y. (2012). Effect of plyometric training on the agility of students enrolled in required College Badminton Programme. Int. J. Appl. Sports Sci. 24, 18-24. doi: 10.24985/ijass.2012.24.1.18

Majeed, K. N. A., and Latheef, M. N. A. (2016). Effects of plyometric training on agility and dynamic postural control in badminton players. Int. J. Sports Sci. Fitness 6, 100-112.

Makhlouf, I., Chaouachi, A., Chaouachi, M., Ben Othman, A., Granacher, U., and Behm, D. G. (2018). Combination of agility and plyometric training provides similar training benefits as combined balance and plyometric training in young soccer players. Front. Physiol. 9:17. doi: 10.3389/fphys.2018.01611

Manouras, N., Papanikolaou, Z., Karatrantou, K., Kouvarakis, P., and Gerodimos, V. (2016). The efficacy of vertical vs. horizontal plyometric training on speed, jumping performance and agility in soccer players. Int. J. Sports Sci. Coach. 11, 702-709. doi: 10.1177/1747954116667108

Manrique, D. C., and Gonzalez-Badillo, J. (2003). Analysis of the characteristics of competitive badminton. Br. J. Sports Med. 37, 62-66. doi: 10.1136/bjsm. 37.1 .62
Markovic, G., and Mikulic, P. (2010). Neuro-musculoskeletal and performance adaptations to lower-extremity plyometric training. Sports Med. 40, 859-895. doi: 10.2165/11318370-000000000-00000

Masu, Y., Muramatsu, K., and Hayashi, N. (2014). Characteristics of sway in the center of gravity of badminton players. J. Phys. Ther. Sci. 26, 1671-1674. doi: $10.1589 /$ jpts.26.1671

Middleton, G., Bishop, D. C., Smith, C., and Gee, T. I. (2016). Effectiveness of a low-frequency sports-specific resistance and plyometric training programme: the case of an elite junior badminton player. Int. J. Coach. Sci. 10, 24-33.

Muehlbauer, T., Wagner, V., Brueckner, D., Schedler, S., Schwiertz, G., Kiss, R., et al. (2019). Effects of a blocked versus an alternated sequence of balance and plyometric training on physical performance in youth soccer players. BMC Sports Sci. Med. Rehabil. 11:18. doi: 10.1186/s13102-019-0131-y

Negra, Y., Chaabene, H., Sammoud, S., Bouguezzi, R., Abbes, M. A., Hachana, Y., et al. (2017a). Effects of plyometric training on physical fitness in prepuberal soccer athletes. Int. J. Sports Med. 38, 370-377. doi: 10.1055/s-0042-122337

Negra, Y., Chaabene, H., Sammoud, S., Bouguezzi, R., Mkaouer, B., Hachana, Y., et al. (2017b). Effects of plyometric training on components of physical fitness in prepuberal male soccer athletes: the role of surface instability. J. Strength Condition. Res. 31, 3295-3304. doi: 10.1519/JSC.0000000000002262

Nonnato, A., Hulton, A. T., Brownlee, T. E., and Beato, M. (2020). The effect of a single session of plyometric training per week on fitness parameters in professional female soccer players: a randomized controlled trial. J. Strength. Cond. Res. doi: 10.1519/JSC.0000000000003591. [Epub ahead of print].

Ozmen, T., and Aydogmus, M. (2016). Effect of core strength training on dynamic balance and agility in adolescent badminton players. J. Bodywork Movement Ther. 20, 565-570. doi: 10.1016/j.jbmt.2015.12.006

Phomsoupha, M., and Laffaye, G. (2015). The science of badminton: game characteristics, anthropometry, physiology, visual fitness and biomechanics. Sports Med. 45, 473-495. doi: 10.1007/s40279-014-0287-2

Rouissi, M., Haddad, M., Bragazzi, N. L., Owen, A. L., Moalla, W., Chtara, M., et al. (2018). Implication of dynamic balance in change of direction performance in young elite soccer players is angle dependent? J. Sports Med. Phys. Fitness 58, 442-449. doi: 10.23736/S0022-4707

Salonikidis, K., and Zafeiridis, A. (2008). The effects of plyometric, tennis-drills, and combined training on reaction, lateral and linear speed, power, and strength in novice tennis players. J. Strength Condition. Res. 22, 182-191. doi: 10.1519/JSC.0b013e31815f57ad

Sekulic, D., Spasic, M., Mirkov, D., Cavar, M., and Sattler, T. (2013). Genderspecific influences of balance, speed, and power on agility performance. $J$. Strength Condition. Res. 27, 802-811. doi: 10.1519/JSC.0b013e31825c2cb0

Shaffer, S. W., Teyhen, D. S., Lorenson, C. L., Warren, R. L., Koreerat, C. M., Straseske, C. A., et al. (2013). Y-balance test: a reliability study involving multiple raters. Military Med. 178, 1264-1270. doi: 10.7205/MILMED-D-13-00222

Tiwari, L., Rai, V., and Srinet, S. (2011). Relationship of selected motor fitness components with the performance of badminton player. Asian J. Phys. Educ. Comput. Sci. Sports 5, 88-91.

Wong, T. K. K., Ma, A. W. W., Liu, K. P. Y., Chung, L. M. Y., Bae, Y. H., Fong, S. S. M., et al. (2019). Balance control, agility, eye-hand coordination, and sport performance of amateur badminton players: a cross-sectional study. Medicine 98:e14134. doi: 10.1097/MD.0000000000014134

Conflict of Interest: The authors declare that the research was conducted in the absence of any commercial or financial relationships that could be construed as a potential conflict of interest.

Copyright (c) 2021 Guo, Huang, Zhou, Leng, Gong, Cui and Bao. This is an openaccess article distributed under the terms of the Creative Commons Attribution License (CC BY). The use, distribution or reproduction in other forums is permitted, provided the original author(s) and the copyright owner(s) are credited and that the original publication in this journal is cited, in accordance with accepted academic practice. No use, distribution or reproduction is permitted which does not comply with these terms. 